Investigación Descriptiva, Correlacional o Cualitativa

Pensar en Movimiento:

Revista de Ciencias del Ejercicio y la Salud ISSN 1659-4436

Vol. 14, No.2, pp. 1- 14

Cierre al 31 de diciembre, 2016

\title{
VARIACIONES EN LA FLEXIBILIDAD DURANTE EL CICLO MENSTRUAL EN DEPORTISTAS UNIVERSITARIAS
}

\section{VARIATIONS IN FLEXIBILITY DURING THE MENSTRUAL CYCLE IN COLLEGE ATHLETES \\ VARIAÇÕES NA FLEXIBILIDADE DURANTE O CICLO MENSTRUAL NAS ESPORTISTAS UNIVERSITÁRIAS}

\author{
Fiorella Moiso Marín, Licda ${ }^{1(A, B, C, D, E)}$ y Andrea Solera Herrera, Ph. D. ${ }^{2,3(B, D, E)}$ \\ moisofiore@gmail.com \\ ${ }^{1}$ Escuela de Terapia Física, Universidad de Costa Rica, Costa Rica \\ ${ }^{2}$ Escuela de Educación Física y Deportes, Universidad de Costa Rica, Costa Rica \\ ${ }^{3}$ Centro de Investigación en Ciencias del Movimiento Humano, \\ Universidad de Costa Rica, Costa Rica \\ Envío original: 03/04/2016. Reenviado: 24/08/2016, 21/10/2016, 14/11/2016. \\ Aceptado: 16/11/2016. Publicado: 21/11/2016. \\ Doi: http://dx.doi.org/10.15517/pensarmov.v14i2.23662
}

\section{RESUMEN}

Moiso, F. y Solera-Herrera, A. (2016). Variaciones en la flexibilidad durante el ciclo menstrual en deportistas universitarias. Pensar en movimiento: Revista de Ciencias del Ejercicio y la Salud, 14(2), 1-14. Con el objetivo de indagar si existen variaciones en la flexibilidad en dos fases del ciclo menstrual (menstrual y ovulatoria) de atletas universitarias, se aplicaron pruebas de flexibilidad funcionales y multiarticulares a 28 mujeres con un promedio de edad de $20.9 \pm$ 3.0 años, nivel de estrés moderado, pocas horas de sueño relacionadas con niveles altos de estrés, y un hábito de consumo de alcohol de al menos una vez al mes en la mayoría de ellas. A nivel general, la $t$ de Student no encontró variaciones significativas en la flexibilidad a lo largo del ciclo menstrual. El nivel de flexibilidad en la prueba de giro de hombros en anteversión fue de $50.73 \pm 17.97^{\circ}$ y de $51.97 \pm 16.76^{\circ}(t=-1.031 ; p=0.312)$, en retroversión fue de $58.14 \pm$ $17.28^{\circ}$ y $60.53 \pm 17.12^{\circ}(t=-1.754 ; p=0.091)$ y en la prueba de "Sit and Reach" fue de $110.63 \pm$ $7.56 \mathrm{~cm}$ y $110.48 \pm 8.31 \mathrm{~cm}(t=0.252 ; p=0.803)$ en la fase ovulatoria y menstrual respectivamente. 
No obstante, la $t$ de Student estratificada, encontró mayor flexibilidad en la fase ovulatoria en mujeres que consumían regularmente alcohol $(p=0.020)$ y tenían un nivel de estrés más alto ( $p=0.040)$ para la prueba de giro de hombros en anteversión, y en mujeres que no dormían bien $(p=0.030)$, dormían menos de 7 horas diarias $(p=0.040)$ o tenían una fase lútea más corta -igual o menor a 9 días- $(p=0.006)$ para la prueba de giro de hombros en retroversión. En conclusión, no hubo variaciones significativas en la flexibilidad a lo largo del ciclo menstrual en la población general. Sin embargo, ciertas condiciones sociodemográficas y clínicas parecieran predisponer a variaciones durante el mismo.

Palabras clave: flexibilidad; ciclo menstrual; deportistas

\begin{abstract}
Moiso, F. y Solera-Herrera, A. (2016). Variations in Flexibility During the Menstrual Cycle in College Athletes. Pensar en Movimiento: Revista de Ciencias del Ejercicio y la Salud, 14(2), 1-14. To inquire whether variations in flexibility in two phases of the menstrual cycle (menstrual and ovulation), tests of flexibility were apply to 28 university women athletes between $20.9 \pm 3.0$ year old with moderate level of stress, few sleeping hours related to high stress levels, and alcohol consumption once a month in the majority of the sample. In general, Student's $t$ did not find significant variations in flexibility during the menstrual cycle. The flexibility level in the test of shoulder turning in ante-version was $50.73 \pm 17.97^{\circ}$ and $51.97 \pm 16.76^{\circ}(t=-1.031 ; p=0.312)$, and the sit and reach flexibility test was $110.63 \pm 7.56 \mathrm{~cm}$ y $110.48 \pm 8.31 \mathrm{~cm}(t=0.252 ; p=$ 0.803 ) during ovulation and menstrual, respectively. However, the stratified Student's t found higher flexibility during ovulation in women who consumed alcohol $(p=0.020)$ and higher stress levels $(p=0.040)$ for the shoulder turning in ante-version, and in women who did not sleep well $(p=0.030)$, or slept less than 7 hours daily $(p=0.040)$, or had a shorter luteal phase, about 9 days, $(p=0.006)$ for the shoulder turning in retroversion. In brief, there were no significant variations in flexibility throughout the menstrual cycle in the sample. Nonetheless, some sociodemographic and clinical conditions may predispose to some variations during the tests.

Key Words: Flexibility, menstrual cycle, athletes
\end{abstract}

\title{
RESUMO
}

Moiso, F. e Solera-Herrera, A. (2016). Variações na flexibilidade durante o ciclo menstrual nas esportistas universitárias. Pensar en movimiento: Revista das Ciências do Exercício e da Saúde, 14(2), 1-14. Com o objetivo de indagar se existem variações na flexibilidade em duas fases do ciclo menstrual (menstrual e ovulatória) de atletas universitárias, aplicaram-se provas de flexibilidade funcionais e multiarticulares em 28 mulheres com uma média de idade de 20.9 \pm 3.0 anos, nível de estresse moderado, poucas horas de sono relacionadas com níveis altos de estresse, e um hábito de consumo de álcool de ao menos uma vez por mês, na maioria delas. Ao nível geral, a $t$ de Student não encontrou variações significativas na flexibilidade ao longo do ciclo menstrual. O nível de flexibilidade na prova de rotação de ombros em anteversão foi de $50.73 \pm 17.97^{\circ}$ e de $51.97 \pm 16.76^{\circ}(t=-1.031 ; p=0.312)$, em retroversão foi de $58.14 \pm$ $17.28^{\circ}$ e $60.53 \pm 17.12^{\circ}(t=-1.754 ; p=0.091)$ e na prova de "Sit and Reach" foi de $110.63 \pm$ $7.56 \mathrm{~cm}$ e $110.48 \pm 8.31 \mathrm{~cm}(t=0.252 ; p=0.803)$ na fase ovulatória e menstrual 
respectivamente. Não obstante, a $t$ de Student estratificada, encontrou maior flexibilidade na fase ovulatória em mulheres que consumiam álcool regularmente $(p=0.020)$ e tinham um nível de estresse mais altos $(p=0.040)$ na prova de rotação de ombros em anteversão, e em mulheres que não dormiam bem $(p=0.030)$, dormiam menos de 7 horas diárias $(p=0.040)$ ou tinham uma fase lútea mais curta - igual ou menos a 9 dias- $(p=0.006)$ na prova de rotação de ombros na retroversão. Enfim, não houve variações significativas na flexibilidade ao longo do ciclo menstrual na população geral. Porém, certas condições sociodemográficas e clínicas pareciam predispor as variações durante o mesmo.

Palavras-chave: flexibilidade; ciclo menstrual; esportistas

Epidemiológicamente, cuando se considera la relación entre el número de lesiones y el número de participantes, el riesgo de sufrir una lesión deportiva es mayor en mujeres (Moreno, Rodríguez \& Seco, 2008). Por esta razón, se ha promovido el estudio de los factores de riesgo, tanto intrínsecos como extrínsecos, que condicionan a un mayor riesgo para ciertas patologías en la población femenina. Uno de estos factores es el ciclo menstrual, puesto que expone a la mujer a múltiples fluctuaciones de las concentraciones de hormonas endógenas en pocos días (Pollard, Braun \& Hamill, 2006).

Cabe aclarar que el ciclo menstrual puede dividirse, en general, en dos fases: fase folicular (que inicia con la menstruación o fase menstrual) y fase lútea (la cual inicia el día posterior al día de ovulación) (Fehring, Schneider \& Raviele, 2006).

Una de las aptitudes físicas que se ha propuesto que puede ser modificada hormonalmente es la flexibilidad, para la cual es importante la elasticidad del músculo, de los tejidos conjuntivos y de los ligamentos (Viana, Rosa, Cardoso \& de Freitas, 1999).

En una revisión bibliográfica sobre las variaciones hormonales y su efecto en la laxitud ligamentosa y la propensión a la lesión del ligamento cruzado anterior (Tabar, 2014), se encontró que las variaciones en las concentraciones de estrógenos y progesterona pueden provocar cambios en la estructura y función del colágeno, lo que consecuentemente influye sobre las propiedades mecánicas de los ligamentos. Estos cambios, dependientes de las concentraciones circulantes de las hormonas, también han sido descritos en los tendones, en donde se ha demostrado que los estrógenos reducen la síntesis de colágeno, incrementan su degradación y contribuyen a una mayor docilidad (Morse, Spencer, Hussain \& Onambele, 2013).

Sin embargo, no hay concordancia en los estudios con respecto a cuál es el momento en el que se da una mayor laxitud articular (Tabar, 2014).

La importancia de conocer la relación entre el ciclo menstrual y la flexibilidad reside en que se ha asociado la laxitud ligamentosa como factor predisponente de lesiones músculoesqueléticas. Esto se fundamenta en que la laxitud articular conduce a hipermovilidad articular y les exige un mayor esfuerzo a las estructuras estabilizadoras tales como ligamentos, cápsulas articulares y músculos (Bin Abd, Bin Ali \& Tet Sen, 2014). Este hecho apunta a la lógica de que, si en un mismo individuo hubiera días de mayor laxitud, habría por tanto días de mayor exposición al riesgo, lo que debería tomarse en cuenta en la actividad deportiva. 
El desconocer este comportamiento puede exponer a las deportistas a realizar movimientos de alta intensidad en los días de mayor laxitud o riesgo, de tal manera que conduce al desarrollo de una lesión física.

Esta relación sería aún más importante para las deportistas con antecedentes de lesiones, dado el mayor riesgo en el que se encuentran de sufrir una nueva lesión (Hägglund, Waldén \& Ekstrand, 2006), ya que el sumarle un factor de riesgo adicional, las expondría hacia una condición más vulnerable. De ser así, convendría que las atletas tomaran en consideración su ciclo menstrual antes de participar en entrenamientos de alta potencia o volumen.

\section{METODOLOGÍA}

Diseño. El presente estudio tuvo un enfoque cuantitativo, observacional analítico y longitudinal prospectivo, y se realizó a lo largo de un ciclo menstrual de las participantes, durante los meses de abril a junio del 2015 en las Instalaciones Deportivas de la Universidad de Costa Rica (UCR).

Participantes. Se trabajó con todas las mujeres integrantes de los equipos deportivos de la UCR que cumplieron con los siguientes criterios de inclusión:

- Tener entre 18 y 30 años de edad.

- Haber tenido la menarquia hace por lo menos dos años.

- Tener un ciclo menstrual regular entre 20 y 36 días.

- Tener ya sea un Índice de Masa Corporal (IMC) entre 16 y 32, o bien un porcentaje de grasa entre $14 \%$ y $35 \%$.

- No utilizar ni haber utilizado anticonceptivos hormonales por los últimos tres meses.

- No tener un diagnóstico de Síndrome de Ovario Poliquístico.

- No tener durante el periodo de la investigación una lesión física que fluctuara importantemente en grado de dolor.

Además, se excluyeron los datos de las participantes en los que las pruebas de flexibilidad de la fase ovulatoria se habían realizado fuera del rango considerado normal según la teoría (de 9 a 20 días) (Cole, Ladner \& Byrn, 2009; Fehring, Schneider \& Raviele, 2006; Mihm, Gangooly \& Mattukrishna, 2011).

De esta forma, la población final comprendió a un total de 28 mujeres.

Instrumentos de medición. Para determinar la fase ovulatoria se recurrió al uso de pruebas de ovulación 'por tira', las cuales son un ensayo inmunocromatográfico in vitro para la determinación cualitativa de la hormona LH en la orina y cuya eficacia en la determinación del pico de LH ha sido descrita por los fabricantes de un 99\% (Wondfo Biotech, 2014).

Se realizó una entrevista a cada participante -en donde se registraron las características sociodemográficas, clínicas y relacionadas al ciclo menstrual- y una valoración física general que incluyó peso, talla, medidas antropométricas, IMC y porcentaje de grasa por medio de un monitor de bioimpedancia eléctrica Omron HBF510LA.

$-4-$

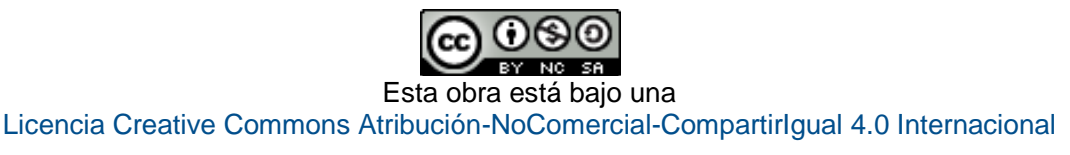


Dada la alta variabilidad de la longitud del ciclo menstrual (Mihm, Gangooly \& Muttukrishna, 2011) se utilizó tanto un cuestionario del ciclo menstrual como un diario menstrual.

Se utilizaron pruebas de flexibilidad multiarticulares, ya que se pretendió estudiar un efecto sumado de varias articulaciones, buscando observar un cambio que, quizás, en el estudio de una articulación no parezca significativo. Estas pruebas poseen un grado confiable de fiabilidad (Ayala, Sainz, de Ste Croix \& Santoja, 2012; Ayala \& Sainz, 2011), los cuales oscilan entre $r=0.91$ y 0.98 (Irurtia, Busquets, Carrasco, Ferrer \& Marina, 2010). Estas pruebas fueron llevadas a cabo por protocolos de medición estandarizados, además de que se ejecutan las modalidades activas en todas las pruebas dado que estas poseen una fiabilidad mayor sobre las pruebas pasivas (Gil \& Zuil, 2011).

Las medidas antropométricas para las pruebas realizadas incluyeron el diámetro biacromial (distancia mínima entre cada acromion) y la longitud de la extremidad superior (distancia mínima entre el acromion y la punta de los dedos). La distancia de separación fue definida como la distancia lineal entre los lados externos de las manos (Irurtia, Busquets, Carrasco, Ferrer \& Marina, 2010).

A continuación su descripción:

Prueba de giro de hombros. En esta prueba, la cual evalúa la flexibilidad en la cintura escapular, se realizaron dos variaciones: la prueba de giro de hombros con bastón en retroversión (PGHR) y la prueba de giro de hombros con bastón en anteversión (PGHA):

Se ejecuta de pie, sujetando con ambas manos un bastón con los brazos en dirección paralela al cuerpo, con los codos en extensión y los brazos en rotación interna.

En la variación en anteversión, la participante sostiene el bastón en la parte anterior del cuerpo y debe llevarlo a la parte posterior pasándolo por encima de la cabeza, con la mínima distancia posible entre las manos. En la variación en retroversión, la participante sostiene el bastón en la parte posterior del cuerpo y debe llevarlo a la parte anterior, igualmente pasándolo por encima de la cabeza y con la mínima distancia posible entre las manos. El tronco debe mantenerse erguido perpendicular al suelo, y las palmas de las manos en contacto con el bastón durante el giro simultáneo de hombros.

El rango de movimiento se calculó por medio de la fórmula descrita por otros investigadores (Irurtia, Busquets, Carrasco, Ferrer \& Marina, 2010):

$$
\alpha_{\text {giro }}=2 \operatorname{arcsen}\left[D s_{\text {giro }}-D b a /(2 L b)\right]
$$

Donde:

$\alpha_{\text {giro }}=$ ángulo de hombros $\left({ }^{\circ}\right)$;

Ds = distancia de separación $(\mathrm{cm})$;

$\mathrm{Dba}=$ diámetro biacromial $(\mathrm{cm})$;

$\mathrm{Lb}=$ longitud de la extremidad superior $(\mathrm{cm})$.

$-5-$

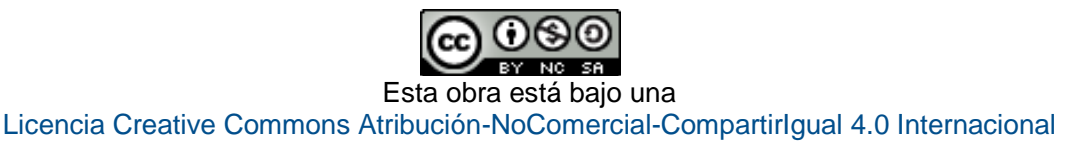


El incremento de los valores de las angulaciones en las pruebas PGHA y PHGR significó una disminución en la flexibilidad, mientras que una disminución significó un aumento de la flexibilidad.

Prueba de "Sit and Reach" (PSR). La PSR evalúa principalmente miembros inferiores y espalda baja (Ayala, Sainz, de Ste Croix \& Santoja, 2012).

Se aplica sentada en el suelo con la cadera en contacto con una pared, las rodillas en extensión y los miembros superiores extendidos con las manos superpuestas una encima de otra. La participante debe intentar flexionar al máximo el tronco sobre los miembros inferiores y alcanzar una distancia máxima entre la punta de los dedos de las manos y la pared. La posición final debe ser mantenida durante 3 segundos.

Para el análisis de los resultados de esta prueba se utilizaron las distancias lineales máximas obtenidas, de modo que una mayor distancia lineal indicó un mayor grado de flexibilidad.

Procedimiento. Primeramente, se les realizó una entrevista y valoración física a las participantes, y se les instruyó en el llenado del diario menstrual y la ejecución de las pruebas de ovulación y las pruebas de flexibilidad.

Las mediciones en la fase menstrual se realizaron en el primero o segundo día de la menstruación, mientras que en la fase ovulatoria se ejecutaron en las siguientes 24 horas del momento en que diera positivo la prueba de ovulación.

Previo a las pruebas de flexibilidad, se realizó un calentamiento estandarizado que incluía correr y ejercicios de estiramiento. Las participantes realizaron cada prueba de flexibilidad 2 veces, cada día de medición en el siguiente orden: PSR, PSR, PGHA, PGHR, PGHA, PGHR. Dado que lo que interesaba conocer era la flexibilidad máxima de las participantes. Se utilizó el valor de flexibilidad máxima para cada prueba, conforme a la metodología seguida por otros investigadores (Irurtia, Busquets, Carrasco, Ferrer \& Marina, 2010).

Para confirmar que la prueba de flexibilidad en la fase ovulatoria se hubiera realizado en el periodo correcto, se realizó una retrospección con la utilización de la información del diario menstrual y la confirmación de que la fase lútea tuviera una longitud entre 7-19 días. Este rango de días se basó en las variabilidades normales que se dan intra e intersujetos (Mihm, Gangooly \& Muttukrishna, 2011; Cole, Ladner \& Byrn, 2009).

Análisis estadístico. Los análisis fueron realizados con el Paquete Estadístico para las Ciencias Sociales (SPSS Inc. Chicago, Illinois, USA) versión 22.0. La significancia estadística se estableció a priori en $p<0.05$.

Para la estadística descriptiva se obtuvieron promedios y desviaciones estándar $(M \pm D E)$, mientras que para la inferencial se realizaron pruebas de t de Student para muestras repetidas. Estos análisis se realizaron de forma global para determinar si existía diferencia entre la flexibilidad presentada en las diferentes fases del ciclo menstrual, y luego de forma estratificada, en donde se consideraron las variables que podrían ser fuente de confusión, como las siguientes: edad, porcentaje de grasa, IMC, condición de hiperlaxitud ligamentosa

$-6-$

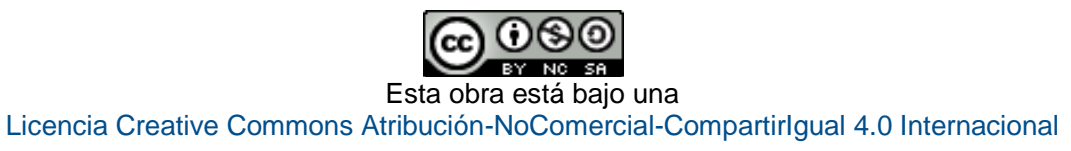


-evaluada con los criterios de Beighton- (Menéndez, 2005), tiempo de entrenamiento de la disciplina deportiva, tiempo de actividad física total semanal, consumo actual de alcohol, nivel de estrés, percepción de dormir bien, número promedio de horas de sueño, incidencia de una lesión de hombro, incidencia de una lesión de espalda, edad de menarquía, número de días de sangrado, presencia de dolor menstrual, uso de medicamentos para el dolor menstrual, incidencia de uso de anticonceptivos hormonales, longitud del ciclo menstrual y longitud de la fase lútea y folicular.

\section{RESULTADOS}

Caracterización de las participantes. En la Tabla 1 se presenta la estadística descriptiva de las características sociodemográficas, clínicas y relacionadas con el ciclo menstrual de la población.

Con respecto a los estilos de vida, ninguna atleta refirió consumir actualmente tabaco o drogas ilícitas, no obstante, la mayoría sí refirió consumir regularmente bebidas alcohólicas, lo más habitual era un consumo mínimo de una vez al mes.

Se encontró un nivel de estrés entre moderado y alto con un promedio de 6.25 según una escala numérica subjetiva del 0 al 10, siendo el valor más común un 8.

Tabla 1

Características sociodemográficas y clínicas relacionadas con el ciclo menstrual

\begin{tabular}{lccc}
\hline Variable & Promedio & Desviación Estándar & Rango \\
\hline Edad (años) & 20.9 & 3.0 & {$[18 ; 30]$} \\
IMC & 22.7 & 3.3 & {$[16.1 ; 31.1]$} \\
Porcentaje de Grasa & 30.6 & 6.5 & {$[14 ; 44.1]$} \\
Longitud del ciclo menstrual (días) & 28.4 & 2.7 & {$[24 ; 3]$} \\
Días de sangrado & 5.0 & 1.2 & {$[3 ; 8]$} \\
Fase folicular (días) & 16.5 & 2.5 & {$[12 ; 21]$} \\
Fase lútea (días) & 11.9 & 2.5 & {$[7 ; 2]$} \\
\hline
\end{tabular}

Fuente: elaboración propia.

Resultados de flexibilidad. La $t$ de Student, en la población general, determinó que no hubo diferencias significativas en la flexibilidad con relación a la fase menstrual para la PGHA. El promedio de flexibilidad en la prueba PGHA fue de $50.73 \pm 17.97^{\circ}$ en la fase ovulatoria y de $51.97 \pm 16.76^{\circ}$ en la fase menstrual $(t=-1.031 ; p=0.312)$.

No obstante, el análisis estratificado indicó diferencias significativas en la población ( $p<$ $0.05)$, en donde se observó un mayor grado de flexibilidad en la fase ovulatoria con respecto a la fase menstrual en las participantes que sí consumían alcohol, las que tenían un nivel de estrés igual o superior a 8, las que tenían una longitud del ciclo menstrual mayor o igual a 30 días y las que tenían una longitud de fase folicular igual o mayor a 17 días (Tabla 2). 
Tabla 2

Factores influyentes en los cambios en la flexibilidad durante el ciclo menstrual según la PGHA

\begin{tabular}{|c|c|c|c|c|c|}
\hline Factores influyentes & Estratos & $\begin{array}{c}\text { Flexibilidad } \\
\text { en la fase } \\
\text { ovulatoria }\left({ }^{\circ}\right)\end{array}$ & $\begin{array}{c}\text { Flexibilidad } \\
\text { en la fase } \\
\text { menstrual }\left(^{\circ}\right)\end{array}$ & $n$ & $\begin{array}{c}t \text { de } \\
\text { Student } \\
p\end{array}$ \\
\hline $\begin{array}{l}\text { Consumo actual de } \\
\text { alcohol }\end{array}$ & $\begin{array}{l}\text { Sí consume } \\
\text { No consume }\end{array}$ & $\begin{array}{l}48.56 \\
54.09\end{array}$ & $\begin{array}{l}52.33 \\
51.42\end{array}$ & $\begin{array}{l}17 \\
11\end{array}$ & $\begin{array}{l}\mathbf{0 . 0 2 0} \\
0.102\end{array}$ \\
\hline Nivel de estrés ${ }^{\dagger}$ & $\begin{array}{l}<\text { a } 8 \\
\geq \text { a } 8\end{array}$ & $\begin{array}{l}51.88 \\
48.32\end{array}$ & $\begin{array}{l}51.44 \\
53.10\end{array}$ & $\begin{array}{c}19 \\
9\end{array}$ & $\begin{array}{l}0.755 \\
\mathbf{0 . 0 4 0}\end{array}$ \\
\hline $\begin{array}{l}\text { Longitud del ciclo } \\
\text { menstrual }\end{array}$ & $\begin{array}{l}<\text { a } 30 \text { días } \\
\geq \text { a } 30 \text { días }\end{array}$ & $\begin{array}{l}48.36 \\
55.00\end{array}$ & $\begin{array}{l}48.38 \\
58.45\end{array}$ & $\begin{array}{l}18 \\
10\end{array}$ & $\begin{array}{l}0.992 \\
\mathbf{0 . 0 2 5}\end{array}$ \\
\hline $\begin{array}{l}\text { Longitud de la fase } \\
\text { folicular }\end{array}$ & $\begin{array}{l}<\text { a } 17 \text { días } \\
\geq \text { a } 17 \text { días }\end{array}$ & $\begin{array}{l}47.67 \\
53.80\end{array}$ & $\begin{array}{l}47.59 \\
56.36\end{array}$ & $\begin{array}{l}14 \\
14\end{array}$ & $\begin{array}{l}0.971 \\
\mathbf{0 . 0 4 7}\end{array}$ \\
\hline
\end{tabular}

Nota: †Según la Escala Numérica del Dolor del 0 al 10. Fuente: elaboración propia.

Con respecto a la PGHR, tampoco se observaron diferencias significativas de la flexibilidad entre las fases del ciclo menstrual en la población general. El promedio de flexibilidad en la prueba PGHR fue de $58.14 \pm 17.28^{\circ}$ en la fase ovulatoria y de $60.53 \pm 17.12^{\circ}$ en la fase menstrual $(t=-1.754 ; p=0.091)$

En el análisis estratificado para la PGHR, se encontró que las mujeres con un IMC menor a 23, las que refirieron no dormir bien, las que dormían menos de 7 horas diarias en promedio, y las que tenían una fase lútea menor a 13 días y/o menor a 9 días tuvieron una mayor flexibilidad en la fase ovulatoria con respecto a la lútea $(p<0.05)$ (Tabla 3 ).

En la PSR el promedio del grado de flexibilidad en centímetros fue prácticamente igual en ambos momentos del ciclo menstrual. El promedio de flexibilidad en la prueba PSR fue de $110.63 \pm 7.56 \mathrm{~cm}$ en la fase ovulatoria y de $110.48 \pm 7.56 \mathrm{~cm}$ en la fase menstrual. Luego de aplicada la prueba $t$ de Student no hubo una diferencia significativa entre ambas fases $(t=$ $0.252 ; p=0.803)$.

Ningún factor del análisis estratificado indicó un mayor grado de flexibilidad en la fase ovulatoria en comparación con la fase menstrual, con la excepción de las variables antropométricas: para un IMC menor a $23(p=0.030)$ y un porcentaje de grasa menor al $30 \%$ $(p=0.010)$, la prueba determinó un mayor grado de flexibilidad en la fase menstrual en comparación con la fase ovulatoria.

$-8-$

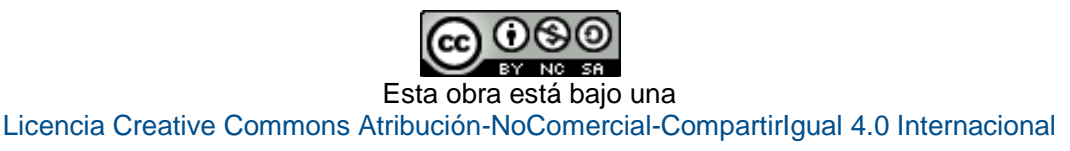


Tabla 3

Factores influyentes en los cambios en la flexibilidad durante el ciclo menstrual según la PGHR

\begin{tabular}{|c|c|c|c|c|c|}
\hline $\begin{array}{l}\text { Factores } \\
\text { influyentes }\end{array}$ & Estratos & $\begin{array}{c}\text { Flexibilidad en } \\
\text { la fase } \\
\text { ovulatoria }\left({ }^{\circ}\right)\end{array}$ & $\begin{array}{l}\text { Flexibilidad } \\
\text { en la fase } \\
\text { menstrual }\left({ }^{\circ}\right)\end{array}$ & $n$ & $\begin{array}{c}t \text { de } \\
\text { Student } \\
p\end{array}$ \\
\hline IMC & $\begin{array}{l}<\text { a } 23 \\
\geq a 23\end{array}$ & $\begin{array}{l}58.42 \\
57.82\end{array}$ & $\begin{array}{l}62.42 \\
58.35\end{array}$ & $\begin{array}{l}15 \\
13\end{array}$ & $\begin{array}{l}\mathbf{0 . 0 4 6} \\
0.794\end{array}$ \\
\hline Calidad de sueño & $\begin{array}{l}\text { No duerme bien } \\
\text { Duerme bien }\end{array}$ & $\begin{array}{l}56.60 \\
58.66\end{array}$ & $\begin{array}{l}63.96 \\
59.38\end{array}$ & $\begin{array}{c}7 \\
21\end{array}$ & $\begin{array}{l}\mathbf{0 . 0 3 0} \\
0.621\end{array}$ \\
\hline Horas de sueño & $\begin{array}{l}<\text { de } 7 \text { horas } \\
7 \text { horas o más }\end{array}$ & $\begin{array}{l}54.45 \\
60.53\end{array}$ & $\begin{array}{l}59.19 \\
61.39\end{array}$ & $\begin{array}{l}11 \\
17\end{array}$ & $\begin{array}{l}\mathbf{0 . 0 4 0} \\
0.633\end{array}$ \\
\hline $\begin{array}{l}\text { Longitud de fase } \\
\text { lútea }\end{array}$ & $\begin{array}{l}\text { < a } 13 \text { días } \\
\geq \text { a } 13 \text { días } \\
\leq \text { o igual a } 9 \text { días } \\
>\text { a } 9 \text { días }\end{array}$ & $\begin{array}{l}57.51 \\
58.87 \\
51.61 \\
59.92\end{array}$ & $\begin{array}{l}61.47 \\
59.44 \\
55.98 \\
61.77\end{array}$ & $\begin{array}{c}15 \\
13 \\
6 \\
22\end{array}$ & $\begin{array}{l}0.030 \\
0.798 \\
0.006 \\
0.291\end{array}$ \\
\hline
\end{tabular}

Fuente: elaboración propia.

\section{DISCUSIÓN}

Con respecto a la caracterización de la población, el nivel de estrés de la población pudo haberse relacionado con un bajo número de horas de sueño, ya que las mujeres que refirieron dormir menos horas tenían en promedio un nivel más alto de estrés.

Las longitudes de fase folicular y lútea se encontraron dentro de los rangos descritos por otros investigadores (Fehring, Schneider \& Raviele, 2006; Mihm, Gangooly \& Muttukrishna, 2011). Sin embargo, se observaron fases foliculares en los límites superiores y fases lúteas en los límites inferiores, lo cual se presume que pudo deberse a la metodología de determinación del día de ovulación o a diferencias reales de las poblaciones estudiadas.

Ahora bien, concerniente a las variaciones en la flexibilidad, primero que todo resulta importante recordar las fluctuaciones normales que se dan en la hormona luteinizante (LH), la hormona folículo estimulante (FSH), los estrógenos y la progesterona, así como al hecho que los niveles bajos de progesterona y los niveles altos de estrógenos se han asociado a una mayor laxitud ligamentosa y, por lo tanto, a una mayor flexibilidad (Prado, 2013).

De esta forma, podemos suponer un relativo equilibrio en la flexibilidad presentado en la fase menstrual -en donde hay bajos niveles tanto de estrógenos como de progesterona-, en comparación con la fase cercana a la ovulación, en donde aumentan los niveles de estrógenos (que aumentarían la flexibilidad) y de progesterona (que disminuiría la flexibilidad). Esto podría explicar la ausencia de una diferencia estadísticamente significativa a nivel de todo el grupo. 
Para la PGHA y según los resultados de la $t$ de Student, el factor que mayor diferencia significativa tuvo fue el consumo actual de alcohol, incluidas las mujeres que lo consumían semanal y mensualmente. La influencia del alcohol sobre la variabilidad de la flexibilidad podría explicarse por los efectos neuroendocrinos que tiene el alcohol, pues este actúa sobre el eje hipotálamo-pituitaria-gónadas, y puede inhibir la liberación de la hormona liberadora de gonadotrofinas $(\mathrm{GnRH})$ e incrementar los niveles de cortisol liberados por la corteza adrenal (Rajendram, Hunter \& Preedy, 2013).

Se ha observado que un consumo elevado de alcohol puede provocar desregulaciones del ciclo menstrual y niveles elevados de estradiol en mujeres, hormona que se libera previo a la ovulación, lo cual podría causar un aumento de la flexibilidad (Prado, 2013). A pesar de que este efecto se ha observado en ingestas altas de etanol y que en el presente estudio no se registró cuantitativamente la exposición a este factor, la vía expuesta constituye un posible mecanismo fisiológico que podría explicar los resultados encontrados.

La influencia del estrés sobre los cambios en la flexibilidad podría deberse a los cambios hormonales que puede provocar este factor (Labad et al., 2015). Cabe aclarar, también, que los niveles de estrés percibido se han relacionado positivamente a través de numerosos estudios con los niveles de hormonas de estrés como epinefrina, norepinefrina y cortisol (Davydov, Shapiro, Goldstein \& Chicz-DeMet, 2005). Niveles altos de dichas hormonas pueden suprimir la secreción de la GnRH, lo que a su vez podría reducir los niveles de LH (León, 2000). Así, podría sugerirse que las mujeres que refirieron un nivel de estrés mayor o igual a 8 podrían haber tenido concentraciones más bajas de LH.

La LH es la hormona que desencadena la ovulación y se relaciona con la transformación del folículo en el cuerpo lúteo, el cual secreta progesterona (León, 2000). Un subdesarrollo luteal, causado por la supresión de la vía mencionada, causaría una menor concentración relativa de progesterona (American Society for Reproductive Medicine, 2015), lo cual reduciría el papel inhibitorio que tiene sobre la laxitud ligamentosa, lo cual a su vez favorecería una mayor flexibilidad.

El hecho de que tanto las mujeres con menor calidad de sueño y menos horas totales de sueño tuvieran una diferencia significativa en la flexibilidad -para la PGHR- puede estar relacionado entre sí, dado que aproximadamente un $63 \%$ de quienes refirieron dormir menos de 7 horas refirieron también dormir mal. El mecanismo por el cual estos elementos podrían influir en la flexibilidad durante el ciclo menstrual puede explicarse por el factor estresante que representa la restricción del sueño, tanto aguda como prolongada (Maggioa, et al., 2013).

Por su parte, la fase lútea menor a 9 días es considerada según la literatura como una fase lútea corta (Harlow, Windham \& Paramsothy, 2013), y puede ser un signo indicativo de insuficiencia del cuerpo lúteo. Esta condición se caracteriza por bajas concentraciones de progesterona.

Cabe aclarar que los niveles hormonales no se registraron en el estudio, por lo que las vías fisiológicas expuestas son una posible explicación teórica de lo que puede haber influido en los resultados, pero faltarían estudios para confirmar las proposiciones expuestas. Sin embargo, un hecho que respaldaría una menor concentración de progesterona en las mujeres con las fases lúteas cortas -tomando como un principio que la progesterona disminuye la 
flexibilidad (Prado, 2013) - es que a lo largo de todo el ciclo menstrual, las mujeres con fases lúteas más cortas tuvieron mayor flexibilidad que las mujeres con fases lúteas más largas, con un promedio de diferencia de $31,73^{\circ}$ en las pruebas de flexibilidad realizadas, una cifra sumamente resaltable.

El comportamiento diferencial presentado en los resultados de la PSR resulta muy interesante, más aún por el hecho de que las mujeres con un IMC menor a 23 tuvieron una mayor flexibilidad en la PGHR en la fase ovulatoria con respecto a la fase menstrual. Esto quiere decir que los resultados de las pruebas de flexibilidad se contradicen para este factor, lo que hace suponer que el IMC no posee un efecto directo sobre la variabilidad de la flexibilidad durante el ciclo menstrual, y que posiblemente existen otras variables de confusión que hayan intervenido en dichos resultados.

Dada la capacidad de síntesis y metabolismo de estrógenos en el tejido graso, los niveles más altos de grasa se relacionan con niveles más altos de estrógenos (Forga, Petrina \& Barvería, 2002; Balderas y otros, 2013). Debido a que los estrógenos se han relacionado como un factor que incrementa la flexibilidad (Prado, 2013), un aumento del tejido graso podría aumentar la flexibilidad general. Sin embargo, el hecho que las mujeres con menores porcentajes de grasa tuvieron en promedio mayor flexibilidad que las mujeres con mayores porcentajes de grasa no concuerda con los razonamientos expuestos. Por ello, se presume que debe haber otros factores de fondo, tales como el volumen de ejercicio o hábitos nutricionales, relacionados con menores porcentajes de grasa, con variaciones en el ciclo menstrual y por ende con los resultados encontrados.

Una limitación del uso de la PSR para los objetivos de este estudio fue la incidencia de dolor lumbar referido por algunas participantes, lo cual pudo haber condicionado negativamente el desempeño en la prueba, sesgando la influencia del ciclo menstrual directamente sobre la flexibilidad.

Otra considerable limitación de los resultados de esta prueba, fue el analizar los datos por medio de distancias lineales y no ángulos, ya que los resultados pudieron haber sido sesgados por diferencias antropométricas.

\section{CONCLUSIONES}

Se concluye entonces que, en la población general de deportistas participantes en el estudio, no hubo variaciones en la flexibilidad a lo largo del ciclo menstrual, sin embargo, se encontraron ciertas condiciones que parecen predisponer a una mayor vulnerabilidad de sufrir variaciones de esta aptitud física durante el ciclo menstrual, tales como consumo de alcohol, nivel de estrés, cantidad y calidad de sueño, duración de la fase lútea y el porcentaje de grasa.

Por ello, se sugiere que, en futuros estudios, se tomen en cuenta dichas condiciones para mayor desarrollo e investigación en torno al tema y como parámetro de inclusión metodológico.

Por otro lado, la prueba de giro de hombros demostró ser relativamente sensible a cambios en la flexibilidad a lo largo del ciclo menstrual, por lo que se estima que el uso de pruebas funcionales multiarticulares es válido para próximas investigaciones. 
Los resultados adquiridos en esta investigación resultan de importancia para la prevención de lesiones y la promoción de la salud. Su valor será de algún modo dependiente del uso práctico que se le dé en planes de entrenamiento y programas de concientización.

\section{REFERENCIAS}

American Society for Reproductive Medicine. (2015). Current clinical irrelevance of luteal phase deficiency: a committee opinion. Fertility and Sterility, 103(4), 27-32. doi: http://dx.doi.org/10.1016/i.fertnstert.2014.12.128

Ayala, F., y Sainz, P. (2011). Fiabilidad absoluta de las pruebas sit and reach modificado y back saber sit and reach para estimar la flexibilidad isquiosural en jugadores de fútbol sala. Apunts Medicina de l'Esport, 46(170), 81-88. doi: http://dx.doi.org/10.1016/j.apunts.2011.01.001

Ayala, F., Sainz, P., de Ste Croix, M., y Santoja, F. (2012). Fiabilidad y validez de las pruebas sit-and-reach: revisión sistemática. Revista Andaluza de Medicina del Deporte, 05(2), 57-66. http://dx.doi.org/10.1016/S1888-7546(12)70010-2

Balderas, A., Muñoz, D., Castro, J., Ramírez, R., Ángeles, W., Flores, M., . . Solano, P. (2013). Porcentaje de adiposidad y su relación con el índice de inmunorreactividad de los receptores hormonales en mujeres mexicanas con cáncer de mama. Nutrición Hospitalaria, 28, 1321-1329. Recuperado de http://www.nutricionhospitalaria. com/pdf/6470.pdf

Bin Abd, H., Bin Ali, N., \& Tet Sen, H. (2014). Generalized ligamentous laxity may be a predisposing factor for musculoskeletal injuries. Journal of Science and Medicine in Sport, 17(5), 474-478. doi: http://dx.doi.org/10.1016/j.jsams.2013.11.001

Cole, L., Ladner, D., \& Byrn, F. (2009). The normal variabilities of the menstrual cycle. Fertility and Sterility, 91(2), 522-527. doi: http://dx.doi.org/10.1016/j.fertnstert.2007.11.073

Davydov, D., Shapiro, D., Goldstein, I., \& Chicz-DeMet, A. (2005). Moods in everyday situations: effects of menstrual cycle, work, and stress hormones. Journal of Psychosomatic Research, 58(4), 343-349. doi: http://dx.doi.org/10.1016/j.jpsychores.2004.11.003

Fehring, R., Schneider, M., \& Raviele, K. (2006). Variability in the Phases of the Menstrual Cycle. Journal of Obstetric, Gynecologic and Neonatal Nursing, 35(3), 376-384. doi: http://dx.doi.org/10.1111/j.1552-6909.2006.00051.x

Forga, L., Petrina, E., y Barbería, J. (2002). Complicaciones de la obesidad. Anales del Sistema Sanitario de Navarra, 25(1), 117-126. Recuperado de http://recyt.fecyt.es/index.php/ASSN/article/view/5494

Gil, M., y Zuil, J. (2011). Fiabilidad y correlación en la evaluación de la movilidad de rodilla mediante goniómetro e inclinómetro. Fisioterapia, 34(2), 73-78. Recuperado de http://www.elsevier.es/es-revista-fisioterapia-146-articulo-fiabilidad-correlacionevaluacion-movilidad-rodilla-S0211563811001908 
Hägglund, M., Waldén, M., \& Ekstrand, J. (2006). Previous injury as a risk Factor for injury in elite Football: A Prospective Study over two consecutive Seasons. British Journal of Sports Medicine, 40(9), 767-772. doi: http://dx.doi.org/10.1136/bjsm.2006.026609

Harlow, S., Windham, G., \& Paramsothy, P. (2013). Menstruation and Menstrual Disorders: The Epidemiology of Menstruation and Menstrual Dysfunction. En Goldman, M.B., Troisi, R. \& Rexrode, K.M (Eds). Women and Health (pp. 163-177). doi: http://dx.doi.org/10.1016/B978-0-12-384978-6.00012-1

Irurtia, A., Busquets, A., Carrasco, M., Ferrer, B., y Marina, M. (2010). Control de la flexibilidad en jóvenes gimnastas de competición mediante el método trigonométrico: un año de seguimiento. Apunts Medicina de l'Esport, 45(168), 235-242. doi: http://dx.doi.org/10.1016/j.apunts.2010.05.003

Labad, J., Stojanovic, A., Montalvo, I., Solé, M., Cabezas, A., Ortega, L., . . Gutiérrez-Zotes, A. (2015). Stress biomarkers as predictors of transition to psychosis in at-risk mental states: Roles for cortisol, prolactin and albumin. Journal of Psychiatric Research, 60, 163-169. doi:http://dx.doi.org/10.1016/j.jpsychires.2014.10.011

León, C. (2000). Influencia del sexo en la práctica deportiva. Biología de la mujer deportista. Arbor, 168(650), 249-263. doi: http://dx.doi.org/10.3989/arbor.2000.i650.968

Maggioa, M., Colizzi, E., Fisichella, A., Valenti, G., Ceresinia, G., Dall'Aglio, E., . . Ceda, G. (2013). Stress hormones, sleep deprivation and cognition in older adults. Maturitas, 76(1), 22-44. doi: http://dx.doi.org/10.1016/j.maturitas.2013.06.006

Menéndez, F. (2005). De la laxitud a la hipermovilidad articular. Revista Cubana de reumatología, 7-8.

Mihm, M., Gangooly, S., \& Muttukrishna, S. (2011). The normal menstrual cycle in women. Animal Reproduction Science, 124(3-4), 229-236. doi: http://dx.doi.org/10.1016/ j.anireprosci.2010.08.030

Moreno, C., Rodríguez, V., y Seco, J. (2008). Epidemiología de las lesiones deportivas. Fisioterapia, 30(1), 40-48. Recuperado de http://www.elsevier.es/es-revista-fisioterapia146-articulo-epidemiologia-las-lesiones-deportivas-S0211563808729547

Morse, C., Spencer, J., Hussain, A., \& Onambele, G. (2013). The effect of the oral contraceptive pill on the passive stiffness of the human gastrocnemius muscle in vivo. Journal of Musculoskeletal Neuronal Interactions, 13(1), 97-104. Recuperado de https://www.ncbi. nlm.nih.gov/pubmed/23445919

Pollard, C., Braun, B., \& Hamill, J. (2006). Influence of gender, estrogen and exercise on anterior knee laxity. Clinical Biomechanics, 21(10), 1060-1066. doi: http://dx.doi.org/10. 1016/j.clinbiomech.2006.07.002

Prado, A. (2013). Influencia del ciclo menstrual en la flexibilidad en natación sincronizada. AGON International Association of Sport Sciences, 3(2), 53-59. Recuperado de https://dialnet.unirioja.es/servlet/articulo?codigo=4727152

Rajendram, R., Hunter, R., \& Preedy, V. (2013). Alcohol: Absorption, Metabolism, and Physiological Effects. En Caballero, B (Ed). Encyclopedia of Human Nutrition (Third Edition), 1, 40-49. doi: http://dx.doi.org/10.1016/b978-0-12-375083-9.00005-2 
Tabar, V. (2014). Revisión: influencia de los cambios hormonales endógenos sobre las lesiones y la laxitud del ligamento cruzado anterior en mujeres deportistas (Tesis de Licenciatura en Fisioterapia). Recuperado de http://academicae.unavarra.es/xmlui/bitstream/ handle/2454/12023/TFGVirginiaTabarLusarreta.pdf?sequence=1\&isAllowed=y

Viana, A., Rosa, M., Cardoso, S., \& de Freitas, S. (1999). Relação do ciclo menstrual com a flexibilidade de quadril de universitárias. Revista Mineira de Educação Física, 7(2), 5259. Recuperado de http://www.revistamineiradeefi.ufv.br/artigos/arquivos/a518e5 da43cdc41e88825af03abab5ea.pdf

Wondfo Biotech. (2014). Prueba de ovulación en orina en un solo paso № de cat. W2-S. Recuperado de http://www.wondfousa.com/pro.asp?id=102

Participación: A- Financiamiento, B- Diseño del estudio, C- Recolección de datos, D- Análisis estadístico e interpretación de resultados, E- Preparación del manuscrito. 